\title{
The design of a new type of irons
}

\author{
Benke Hao \\ School of North China Electric Power University,Baoding 071000,China \\ 1103430089@qq.com
}

\begin{abstract}
Overhead transmission line maintenance, testing and inspection work part, all need to climb to the top of the pole, and irons is one of the essential tools for climbing pole. At present, some traditional irons safety problems in construction safety is more and more attention, it is bound to improve the existing problems of traditional irons. At present, the main problems are the traditional irons and foot pedal easy to slip, traditional irons do not wear comfortable. In view of these two questions, I proposed the plan that using the lock shoes to tread the structure to carry on the transformation to the pedal part. The scheme achieves the expected goal, and solves the problems of easy slipping and wearing uncomfortable.
\end{abstract}

Key words: Irons, pole shoes, lock.

\section{Background}

It is commonly used in the distribution network operation and maintenance personnel climbing pole working tool, which is composed of a movable hook, buckle body, top tread plate buckle, buckle and non skid rubber pad. You can adjust the size according to the thickness of irons wire rod for different thickness. Irons has the advantages of convenient carrying, on the rod and the lower rod speed. But for the traditional climbers, it is the poor safety used in rainy days. With the standardization management of power supply enterprises, safety management has become the top priority of enterprise management. As one of the essential tools for climbers climbing power lines, the safety management in enterprises has become the focus of concern.

In a small range of power installation company investigation and statistics found that the company nearly 150 front-line operations personnel, 60\% had at least one irons abscission events within a year, $20 \%$ had two or more feet within a year off event. The pole in the process of irons off events, has become one of the reasons can not be ignored in the power accidents.

\section{Research status}

Chuandong Jiang invented a climber anti off foot laces ${ }^{[1]}$, as shown in Figure 1. The structure of soft material constraints of our feet, but this it has certain requirements on the type of shoes, especially shoes on the next big small, the relative position and the structure is not fixed completely and foot pedal effort in the pole process slightly. 

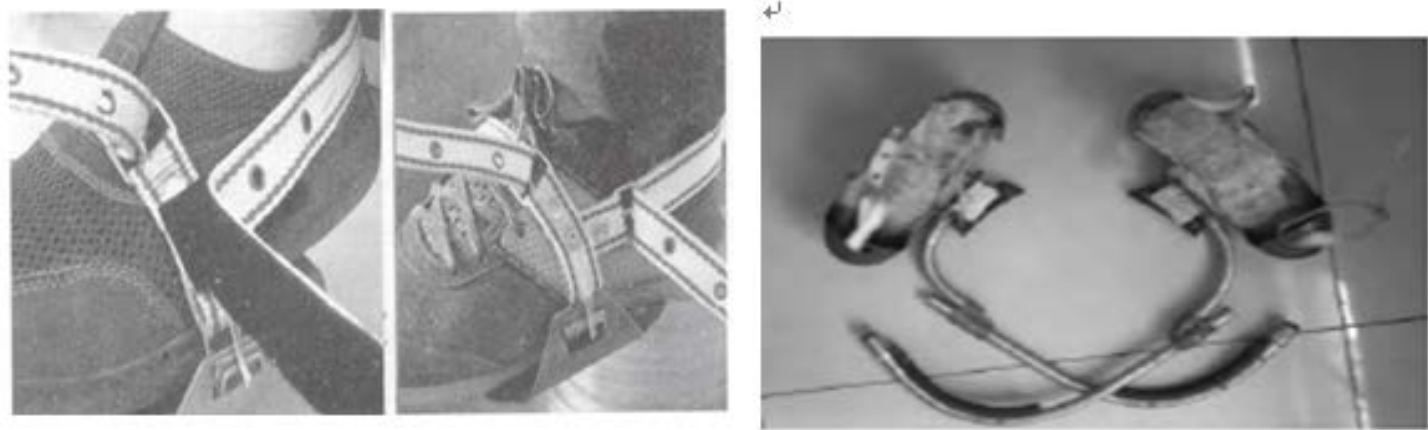

Figure $1 \mathrm{~A}$ kind of anti off foot shoe irons ${ }^{[1]}$ Figure 2 Anti fatigue assembly slip irons Yuan Tang proposed a new anti slip clip irons ${ }^{[2]}$. The anti slip clip in rainy day and wet weather to increase the friction between the irons and poles of the role. Yahui Zheng proposed a skidproof climbers anti fatigue assembly ${ }^{[3]}$,as shown in Figure 2. The climbers will directly weld pedal toe and heel on labor, the "iron irons pedal shoes", directly to the foot cover in the iron shoes, and with fixed, can be realized and the fixed foot feet. The utility model has the advantages that the structure can effectively prevent the skid, but the structure is only suitable for a certain length of shoes

In addition, there are a variety of structures, such as the new structure shown in Figure 3. The structure of the transformation of feet pedal and nylon belt, developing original big foot pedal irons into imitation type on the narrow width of the pedal structure, and the nylon belt of nylon belt back out and forming a ring adjustable size to fix the heel. As shown in Figure 3,the new structure is relatively mature at present, and it is simple and convenient, low cost, and no change in innovation based on the structure of irons is comfortable and convenient. But there are still some deficiencies. First, the nylon belt can not realize the stepless adjustment. Second, although the structure effectively fixes the heel part, but to climb the pole still cannot be fixed feet and feet pedal, unable to effectively lift the pedal rod climbing arduous process.
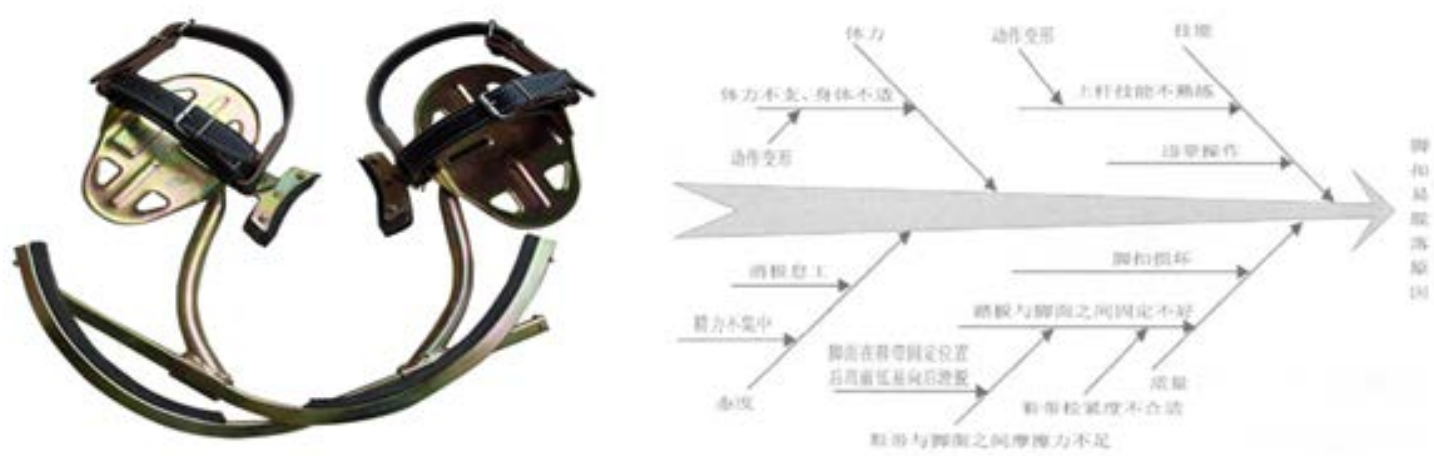

Figure 3 Novel belt structure

Figure 4 The shortcomings of the traditional irons

\section{The analysis of the traditional irons}

Before the design of a new type of climbers, we need to carefully analyze all kinds of 
existing problems in traditional irons.First of all, it is the need to analyze the problem of the sliding feet.This problem is divided into two parts, one part is the slip between the irons and the wire pole; the other part is the slip between the foot and the foot.In addition, it is need to consider the problem of irons comfort, because comfort plays a very important role in safety problems in irons.

\subsection{Irons slip problem}

As shown in Figure 4, there are four main reasons leading to falling irons: attitude, physical strength, skill and quality. We can also see that because the nylon belt tightness can lead to inappropriate climbers fall, this is not because some irons stepless regulation. In the design of a new type of irons,we can put nylon belt regulation set for stepless adjustment, because stepless adjustment relative to the level adjustment has stronger adaptability.We can also see that the shortage of skills will lead to falling irons.The fundamental reason for the lack of skills is because the foot to foot positioning and angle is insufficient, resulting in the construction of the foot has great flexibility. This flexibility will result in the process of the foot pedal pole will be relatively easy in the relative displacement will climb higher and lift the feet foot and foot from the place of the accident.

\subsection{Irons comfort}

After analyzed, the main reason of uncomfortable wearing is single-layer nylon belt.The only place the original irons and instep with the rising need to adjust the relative position to the instep and nylon belt with the foot "take up" so as to realize the pole foot problem.The main solutions are as follows:the one,achieving the foot and foot positioning and clamping, as far as possible to reduce the foot on the foot of freedom.The other,using soft material to connect the foot and foot, so that the construction of the upper and soft materials to enhance the comfort.

\section{The illustration of new irons}

The scheme is based on the following objectives.First,it cannot change the basic structure.Second,it has prevent irons and foot slip function.third, it can improve the construction of comfortable experience during the construction. The last, it considering the urgent need to design process from the program.

Based on the above four points, we searched for the data and finally established the plan. The physical model is shown in the following figure:

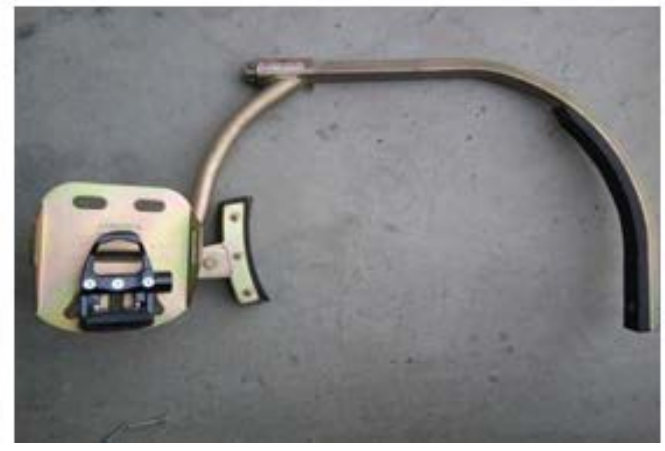

Figure 5 Top view of new irons

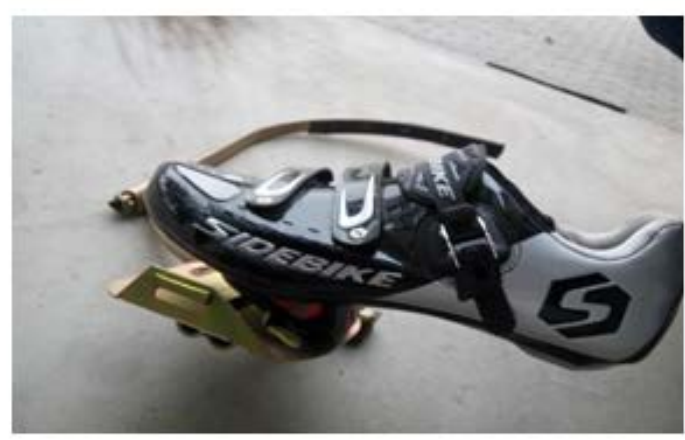

Figure 6The new irons left view 
Figure 5 and figure 6, the new lock lock step irons from shoe structure with inclined countersunk head screws will lock in place of the foot pedal.The utility model only needs to wear a lock shoe which is provided with a locking piece, and the locking piece is buckled in the lock step when wearing.A spring clamping device is arranged at the tail part of the locking step, and when the locking piece is squeezed into the lock step, the spring device is opened, and when the locking piece reaches the corresponding position of the locking step, the spring device is clamped, so that the connection between the locking step and the lock shoe can be realized.

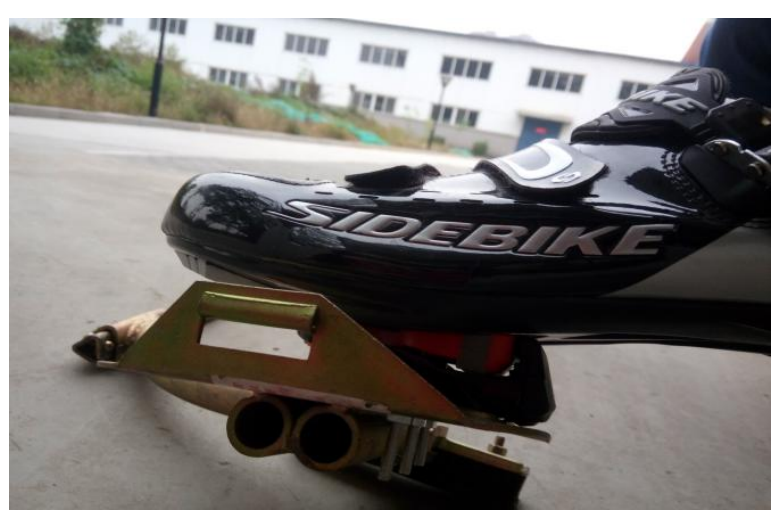

Figure 7 New irons details

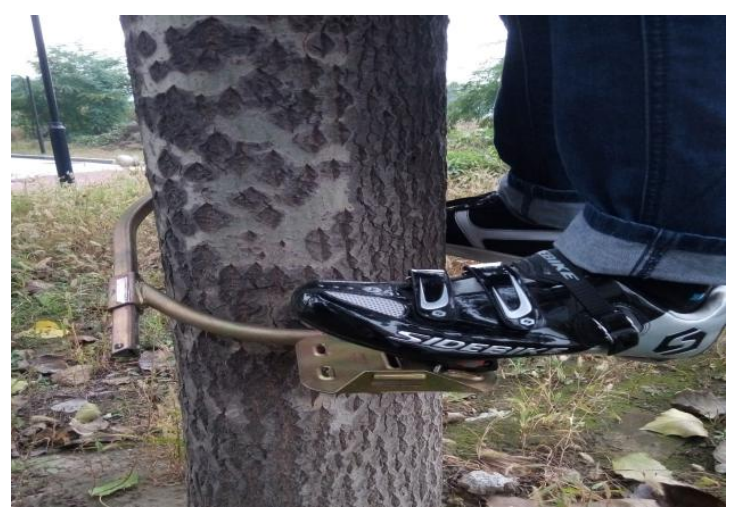

Figure 8 Security verification

As shown in Figure 7, the bottom part of the lock shoe is a locking plate.The bottom of the lock shoe is provided with a polygonal concave hole which is matched with the lock piece.The pits are distributed on the three line of triangular screw hole, which corresponding to the lock plate three through hole.The lock piece by the polygon pits and lock shoes positioning, and then realize the clamping lock plate and the lock shoe by a threaded connection.

As shown in Figure 8, after the establishment of the program, we carried out the corresponding climb experiment, found in the process of climbing more effort.In the scheme, the foot is directly matched with the lock shoe, and the wearing comfort is ensured because the locking shoe has the characteristics of the shoe and the inner lining is a soft material.In addition, in the process of rising, the shoes can be at any time with the foot movement, make the operation more convenient.At the same time, it reduces greatly due to its workers attitude and technical reasons irons off accident. When the separation of emergency requires immediate foot and irons ,The construction needs to outward side direction force will draw on the lock piece from the lock in the pits.

\section{Conclusions}

In summary, we believe that the scheme perfectly achieved our goal, because it doesn't change the original structure but also to avoid the irons slipping accidents maximally and improve the foot in the construction process of comfort, but also to consider from the foot and irons emergency occurs. 


\section{Reference}

[1] Chuandong Jiang, Qingqiang Lian . Irons pedal anti off foot device of [J]. rural electrification, 2013, (02): 40-42.

[2]Yuan Tang. New irons anti slip clip the research and application of [J]. China high-tech enterprises, 2016, (32): 40-41.

[3], Yahui Zheng, Wenlin Zheng. An illumination function [J]. irons rural electrician, 2015, (06): 23. 